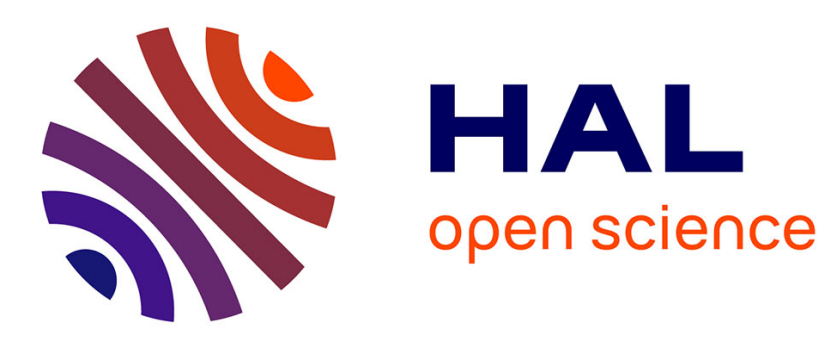

\title{
The characterization of ice cloud properties from Doppler radar measurements.
}

Julien Delanoë, Alain Protat, Dominique Bouniol, A. J. Heymsfield, A. Bansemer, P. R. Brown

\section{To cite this version:}

Julien Delanoë, Alain Protat, Dominique Bouniol, A. J. Heymsfield, A. Bansemer, et al.. The characterization of ice cloud properties from Doppler radar measurements.. Journal of Applied Meteorology and Climatology, 2007, 46 (10), pp.1682-1698. 10.1175/JAM2543.1 . hal-00159848

\section{HAL Id: hal-00159848 \\ https://hal.science/hal-00159848}

Submitted on 29 Nov 2020

HAL is a multi-disciplinary open access archive for the deposit and dissemination of scientific research documents, whether they are published or not. The documents may come from teaching and research institutions in France or abroad, or from public or private research centers.
L'archive ouverte pluridisciplinaire HAL, est destinée au dépôt et à la diffusion de documents scientifiques de niveau recherche, publiés ou non, émanant des établissements d'enseignement et de recherche français ou étrangers, des laboratoires publics ou privés. 


\title{
The Characterization of Ice Cloud Properties from Doppler Radar Measurements
}

\author{
Julien Delanoë \\ Centre d'Etude des Environnements Terrestre et Planétaires, Vélizy-Villacoublay, France, and Department of Meteorology, University \\ of Reading, Reading, United Kingdom
}

\section{A. Protat and D. Bouniol}

Centre d'Etude des Environnements Terrestre et Planétaires, Vélizy-Villacoublay, France

Andrew Heymsfield AND AARON BANSEMer

National Center for Atmospheric Research,* Boulder, Colorado

PHILIP BROwN

Met Office, Exeter, United Kingdom

(Manuscript received 9 June 2006, in final form 24 October 2006)

\begin{abstract}
The paper describes an original method that is complementary to the radar-lidar algorithm method to characterize ice cloud properties. The method makes use of two measurements from a Doppler cloud radar ( 35 or $95 \mathrm{GHz}$ ), namely, the radar reflectivity and the Doppler velocity, to recover the effective radius of crystals, the terminal fall velocity of hydrometeors, the ice water content, and the visible extinction from which the optical depth can be estimated. This radar method relies on the concept of scaling the ice particle size distribution. An error analysis using an extensive in situ airborne microphysical database shows that the expected errors on ice water content and extinction are around $30 \%-40 \%$ and $60 \%$, respectively, including both a calibration error and a bias on the terminal fall velocity of the particles, which all translate into errors in the retrieval of the density-diameter and area-diameter relationships. Comparisons with the radar-lidar method in areas sampled by the two instruments also demonstrate the accuracy of this new method for retrieval of the cloud properties, with a roughly unbiased estimate of all cloud properties with respect to the radar-lidar method. This method is being systematically applied to the cloud radar measurements collected over the three-instrumented sites of the European Cloudnet project to validate the representation of ice clouds in numerical weather prediction models and to build a cloud climatology.
\end{abstract}

\section{Introduction}

The knowledge of the cloud properties has been recently identified as a mandatory step to reach if the operational weather and climate change forecasts are to be improved (Stephens et al. 2002; Stephens 2005). In

* The National Center for Atmospheric Research is sponsored by the National Science Foundation.

Corresponding author address: Julien Delanoë, Department of Meteorology, University of Reading, Earley Gate, P.O. Box 243, Reading RG6 6BB, United Kingdom.

E-mail: j.m.e.delanoe@reading.ac.uk

DOI: $10.1175 / J A M 2543.1$

(C) 2007 American Meteorological Society the framework of the space missions devoted to monitoring the microphysical, radiative, and dynamic properties of clouds at a global scale using cloud radar and lidar combinations [CloudSat/Cloud-Aerosol Lidar and Infrared Pathfinder Satellite Observations (CALIPSO), part of the Afternoon Train ${ }^{1}$, Stephens et al. (2002], there is a need for ground-based and airborne validation of the radar-lidar measurements and products from these space missions. The synergy between radar and lidar instruments (ground based, airborne, and spaceborne) is such that in moderately thick clouds, the

\footnotetext{
${ }^{1}$ The Afternoon Train or "A Train" is the nickname given to a group of satellites that fly close together and pass over the equator in the early afternoon.
} 
liquid/ice water content (IWC) and effective radius of droplets/crystals can be accurately retrieved from radar and lidar measurements (Tinel et al. 2005; Wang and Sassen 2002; Donovan and van Lammeren 2001; Okamoto et al. 2000). The domain of application of the radar-lidar synergy is however limited to a given range of clouds (optical thickness less than 3, roughly). As an example, prefrontal and mixed-phase clouds, which are very common in midlatitude regions, are generally not fully traversed by the lidar. In the present paper we therefore propose an original method complementary to the radar-lidar algorithm, which makes use of the two measurements of a Doppler cloud radar (35 or 95 $\mathrm{GHz}$ ), namely, the radar reflectivity and the Doppler velocity, in order to recover the effective radius and terminal fall velocity of crystals, the ice water content, and the visible extinction, and therefore the visible optical depth. Previously, Matrosov et al. (2002) proposed a method combining Doppler velocity and reflectivity assuming the Rayleigh scattering. Such a method is not really applicable to a radar at $95 \mathrm{GHz}$ when the Rayleigh approximation is no longer valid. In the present paper, we describe a new Doppler radar method available for radars from 3 to $95 \mathrm{GHz}$ (but these could be extended to higher frequencies if needed). Furthermore, it is noteworthy that in the longer term this method might be applied to the Doppler radar of Earth Cloud Aerosol and Radiation Explorer (EarthCARE; European Space Agency 2004).

This radar retrieval method is described in section 3 . It relies on a set of relationships between the cloud properties and the radar measurements, scaled by the intercept parameter of the normalized particle size distribution (Delanoë et al. 2005), the principle of which is described in section 2. These relationships are derived from the different moments of the normalized particle size distribution. The performance of the method is then evaluated in section 4 using a large microphysics database and comparisons with a radar-lidar method. We also conducted an analysis of the sensitivity of the retrieval to the Doppler velocity averaging period. Conclusions are given in section 5 .

\section{General background}

\section{a. The particle size distribution}

Both the two radar observables and the microphysical and radiative parameters that are required to document the cloud properties are tightly linked through the particle size distribution (PSD) in the radar volume. It is well known that the PSD is highly variable in ice clouds, owing to variations over 3-4 decades of the ice water content in a single cloud, and the different ranges of diameters encountered from one cloud to another. However, it has been shown recently that a scaling of the PSD by a normalized parameter $\left(N_{0}^{*}\right)$ significantly reduced this variability (Delanoë et al. 2005; the main results of this paper are summarized in the appendix). In the present study, the PSD is made independent from the ice water content and the mean volumeweighted diameter [see Delanoë et al. (2005) for further details]. A general expression of the PSD can be written as follows:

$$
N\left(D_{\mathrm{eq}}\right)=N_{0}^{*} F\left(D_{\mathrm{eq}} / D_{m}\right),
$$

where $N\left(D_{\text {eq }}\right)$ is the PSD, $F\left(D_{\text {eq }} / D_{m}\right)$ is the normalized PSD, $D_{\text {eq }}$ is the "equivalent melted" diameter (which is the diameter the ice particle would have if it was a spherical water particle of the same mass), and $N_{0}^{*}$ $\left(\mathrm{m}^{-4}\right)$ is the intercept parameter of the PSD proportional to IWC/ $D_{m}^{4}$. The volume-weighted diameter $D_{m}$ is the ratio of the fourth to the third moment of the PSD.

The relationship between the maximum diameter of an ice crystal and its equivalent melted diameter involves an assumption on the ice crystal density, which is a critical point for all-radar and radar-lidar methods (Delanoë et al. 2005). The way this assumption is dealt with in the present radar method has been discussed in Delanoë et al. (2005).

In the same paper, Delanoë et al. (2005) have investigated the stability of the normalized PSD shape in ice clouds, using a very extensive airborne in situ microphysics dataset, including different types of ice clouds, and both midlatitude [1998 Cloud Lidar and Radar Experiment (CLARE98), 1999 Cloud by Ground-Based and Airborne Radar and Lidar (CARL99), European Clouds and Radiation Experiment (EUCREX), Fronts and Atlantic Storm Track Experiment (FASTEX), Atmospheric Radiation Measurement (ARM) Intensive Observation Period (IOP)] and tropical [the Central Equatorial Pacific Experiment (CEPEX) and the Cirrus Regional Study of Tropical Anvils and Cirrus Layers Florida-Area Cirrus Experiment (CRYSTALFACE)] campaigns. They found that as in the case of the raindrop size distribution the normalized PSD was fairly identical from one cloud to another, and therefore proposed to use a single analytical formulation (the so-called modified gamma shape) to describe scaled ice cloud PSDs. Delanoë et al. (2005) have shown that the mean relative error on each cloud and observable parameter is clearly minimized when using the modified gamma shape, leading to biases of less than 3\% for all clouds and instrumental parameters.

This stability in the shape of the normalized PSD of ice clouds is an important result, because it also implies 
that a moment $X$ of the normalized PSD (i.e., IWC/N*, $\left.\alpha / N_{0}^{*}, Z / N_{0}^{*}\right)$ can be related to any moment $Y$ by a power-law relationship $Y=a X^{b}$. When assuming a modified gamma shape, the equivalent $n$th moment of the normalized PSD can be analytically determined.

\section{b. Cloud parameters and radar observables}

The general expressions of the PSD moments can be used to relate the cloud parameters (ice water content, visible extinction, effective radius, ice fall speed, number concentration) and the radar observables (reflectivity, Doppler velocity and spectral width) through statistical relationships. For instance, the radar reflectivity $Z$ (assuming Rayleigh scattering) and ice water content are proportional to the sixth and third equivalent moments of the PSD, respectively. Contrary to the case of Rayleigh scattering, the reflectivity coefficient is however not directly proportional to a moment of the PSD in the case of Mie scattering,

$$
Z_{e}=\frac{\lambda^{4}}{\left|K_{w}\right|^{2} \pi^{5}} 10^{18} \int N(D) \sigma_{\mathrm{bsc}}(\rho, D, \lambda) d D\left(\mathrm{~mm}^{6} \mathrm{~m}^{-3}\right)
$$

where $D$ is the maximum (unmelted) diameter, $\left|K_{w}\right|^{2}=$ 0.93 at $3 \mathrm{GHz}$, and $\sigma_{\mathrm{bsc}}$ is the Mie backscattering coefficient depending on the maximal diameter; $\lambda$ is the wavelength and $\rho$, the density-diameter relationship, can be expressed as

$$
\rho=a_{\rho} D^{b},
$$

where $D$ is in centimeters and $\rho$ is in grams per cubic centimeter.

Here, $a_{\rho}$ can be expressed as a function of $b_{\rho}$ and the limit diameter $D_{1}: a_{\rho}=0.917 /\left(D_{1} \times 10^{4}\right)^{b_{\rho}}$, where $D_{1}$ $(\mu \mathrm{m})$ is the "limit diameter." The rationale for this limit diameter is that for small diameters the ice density exceeds that of the solid ice, which cannot happen in the real world. When the solid ice density is reached the corresponding diameter is the limit diameter, and below this limit diameter the ice density is set at that of solid ice $\left(\rho_{i}=0.917 \mathrm{~g} \mathrm{~cm}^{-3}\right)$.

In the $N_{0}^{*}$ formalism $Z_{e}$ is rewritten as

$Z_{e}=\frac{\lambda^{4}}{\left|K_{w}\right|^{2} \pi^{5}} 10^{18} N_{0}^{*} \int F\left(D / D_{m}\right) \sigma_{\mathrm{bsc}}(\rho, D, \lambda) d D\left(\mathrm{~mm}^{6} \mathrm{~m}^{-3}\right)$,

where $F\left(D / D_{m}\right)$ is the shape of the normalized PSD.

The reflectivity-weighted velocity of a population of ice crystals described by a given PSD can be expressed as

$$
V_{t}=\frac{\int v(D) \sigma_{\mathrm{bsc}}(\rho, D, \lambda) F\left(D / D_{m}\right) d D}{\int \sigma_{\mathrm{bsc}}(\rho, D, \lambda) F\left(D / D_{m}\right) d D}\left(\mathrm{~m} \mathrm{~s}^{-1}\right),
$$

where $v(D)$ is the terminal fall velocity for an ice particle with the diameter $D$. The $v(D)$ relationship is related to the ice density and cross-sectional area [cf. Eqs. (6) and (7)] assumptions (Khvorostyanov and Curry 2002; Mitchell et Heymsfield 2005). The terminal fall velocity for each diameter is derived from the formulation of Mitchell and Heymsfield (2005),

$$
v(D)=\frac{a_{d}(D) \nu}{D}\left(\frac{2 g D^{2}}{\rho_{a} \nu^{2}}\right)^{b_{d}(D)}\left[\frac{m(D)}{A(D)}\right]^{b_{d}(D)}\left(\mathrm{cm} \mathrm{s}^{-1}\right),
$$

where $g$ is the gravitational acceleration, $\rho_{a}$ is the air density, $\nu$ is the kinematic viscosity, and $a_{d}(D)$ and $b_{d}(D)$ are the coefficients of the relationship between $X$ [Best (also called Davies) number] and the Reynolds number, respectively. These coefficients are calculated using Mitchell and Heymsfield (2005),

$$
A=\gamma D^{\sigma}
$$

where $D$ is in centimeters and $A$ is in inverse centimeters squared.

The IWC can be analytically expressed as a function of $N_{0}^{*}$ and $D_{m}$ and the density of liquid water $\rho_{w}$,

$$
\mathrm{IWC}=\frac{N_{0}^{*} \pi \rho_{w} D_{m}}{4^{4}}\left(\mathrm{~g} \mathrm{~cm}^{-3}\right) .
$$

In the geometric optics approximation, the visible extinction coefficient $\alpha$ is proportional to the $\sigma$ th moment of the physical PSD, where $\sigma$ is the exponent of the area-diameter relationship,

$$
\alpha=2 \int N(D) \gamma D^{\sigma} d D\left(\mathrm{~m}^{-1}\right) .
$$

The effective radius $r_{e}$ can be defined as the ratio of the third to the second equivalent moment of the PSD. This translates into a direct analytical relationship between $r_{e}$ and $\alpha$,

$$
r_{e}=\frac{3(\mathrm{IWC})}{2 \rho_{i} \alpha} 10^{6}(\mu \mathrm{m}),
$$

where $\rho_{i}$ is the density of solid ice $\left(0.917 \mathrm{~g} \mathrm{~cm}^{-3}\right)$, IWC is in grams per cubic centimeter, and $\alpha$ is in inverse meters.

All of these parameters depend on the densitydiameter and area-diameter relationships, so the choice of these parameterizations is crucial. For instance, we obtain a bias of about $50 \%$ (standard devia- 

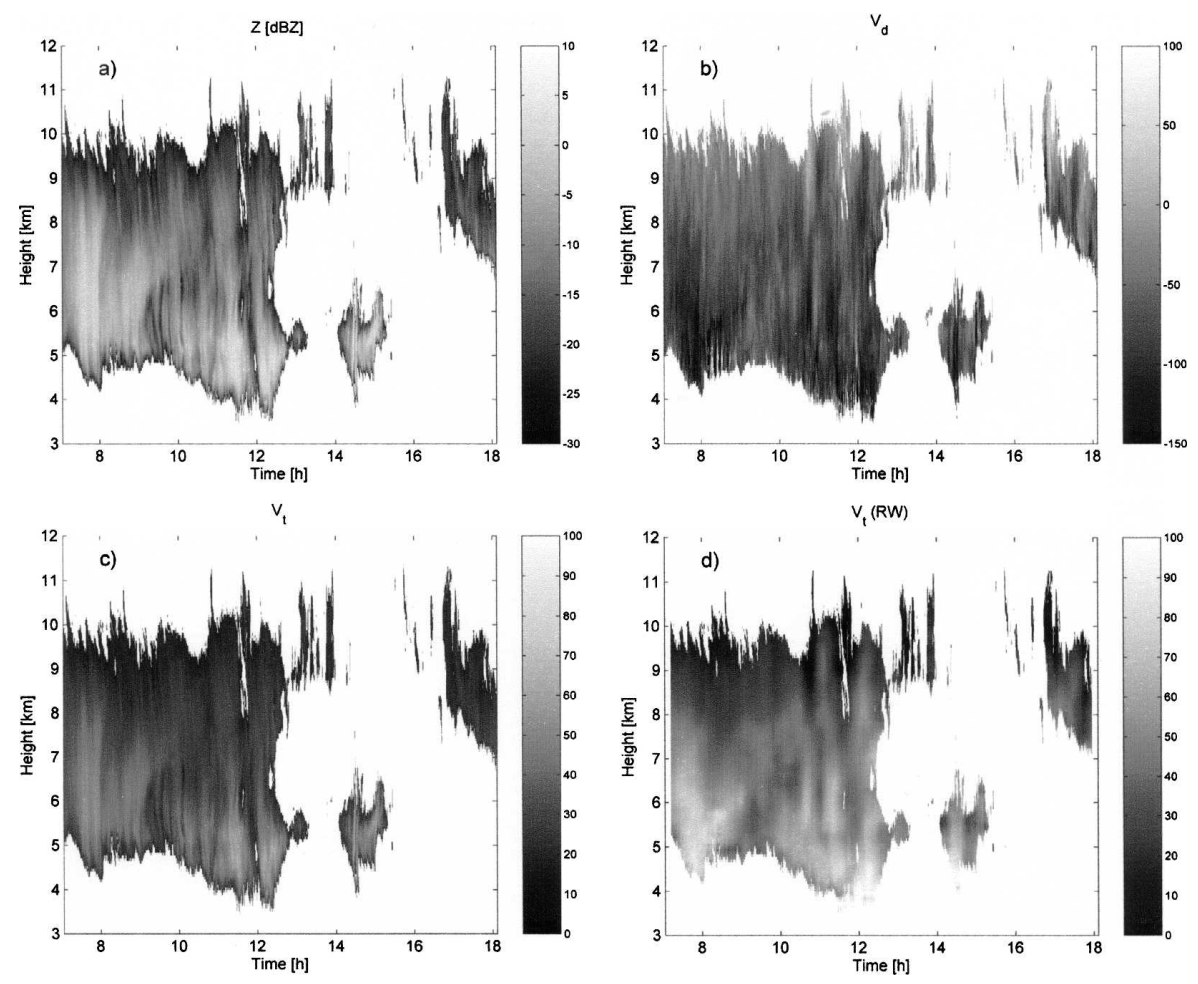

FIG. 1. Flowchart of the RadOn method. The method is described step by step in section 3 .

tion 5\%) between IWC computed in using the Brown and Francis (1995) aggregate density-diameter relationships ( $\rho=0.07 D^{-1.1}$, with $D$ in millimeters) and the relationship for aggregates proposed by Mitchell (1996). For $\alpha$, the bias can reach $36 \%$ (standard deviation $9 \%$ ).

In conclusion, if we can estimate the ice density $N_{0}^{*}$ and $D_{m}$, then we can access an extensive documentation of the ice cloud properties, including ice water content, effective radius, visible extinction (and cloud optical depth), and number concentration. The method proposed in this paper, denoted as radar only (RadOn) herein, consists in estimating these quantities from two radar measurements (radar reflectivity and Doppler velocity). This method is described in the next section.

\section{Principle of the method}

The different steps of the method (summarized on the flowchart of Fig. 1) are described in detail in the following sections.

\section{a. Terminal fall velocity retrieval from Doppler velocity}

Doppler cloud radar does not measure the terminal fall velocity directly; the Doppler measurement is the sum of the reflectivity-weighted velocity of the ice particles $V_{t}$ and the vertical air motion $w$. To estimate $V_{t}$, a statistical approach (hereinafter referred to as the $V_{t}-Z$ approach) has been recently proposed in the case of frontal cyclones and nonprecipitating ice clouds (Protat et al. 2003). It consists of developing statistical relationships between terminal fall velocity and radar reflectivity, which can be expressed as

$$
V_{t}=a Z^{b}\left(\mathrm{~m} \mathrm{~s}^{-1}\right),
$$

where $Z$ is in millimeters to the sixth power per cubic meter and $a$ and $b$ are the coefficients of $V_{t}-Z$ relationship obtained by linear regression.

Within nonprecipitating clouds (i.e., clouds that do not produce precipitation at the ground), the vertical air motions are generally small, even at small scales of motion, as opposed to the case of convective systems. In any case, however, the vertical air motions are not negligible with respect to the terminal fall speed. For a long time span, however (from one to a few hours), the mean vertical air motion should vanish with respect to the mean terminal fall speed, which is much less fluctuating. A statistical power-law relationship between the terminal fall speed and radar reflectivity may therefore be derived from this statistical approach. Figure 2 shows a scatterplot between Doppler velocity and reflectivity from which a $V_{t}-Z$ relationship is derived for a given cloud. Following this approach the scatter around the fitted curve is attributed to the vertical wind 


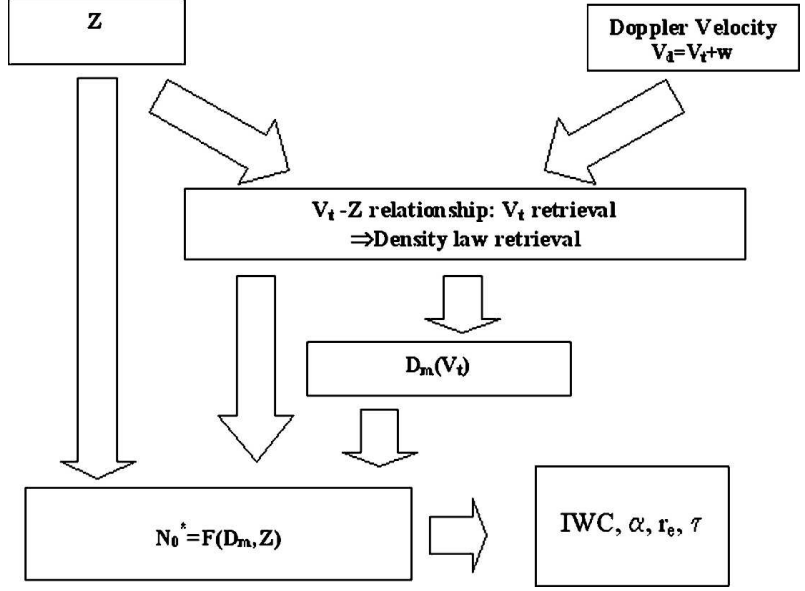

FIG. 2. Doppler velocity as a function of reflectivity measured by the vertically pointing $95-\mathrm{GHz}$ radar on 14 Apr 2003 over the SIRTA instrumented site, in Palaiseau, France. The curve represents the power-law relationship $\left(V_{t}=73.2 Z^{0.2463}\right.$; units for $Z$ : $\mathrm{mm}^{6} \mathrm{~m}^{-3}$ ) derived from the scatterplot.

component only. Figure $3 \mathrm{c}$ shows the corresponding time-height section of $V_{t}$ when this relationship is applied to the time-height cross section of reflectivity.

The implicit assumption of this approach is that the cloud microphysical characteristics do not change within the cloud (the nature of the $V_{t}-Z$ relationship, e.g.). It is clear that this approach is not perfect; in particular, it is expected that the $V_{t}-Z$ relationship will probably change in the vertical, especially for thick ice clouds, for which aggregation will produce a diminution of crystal densities and number.

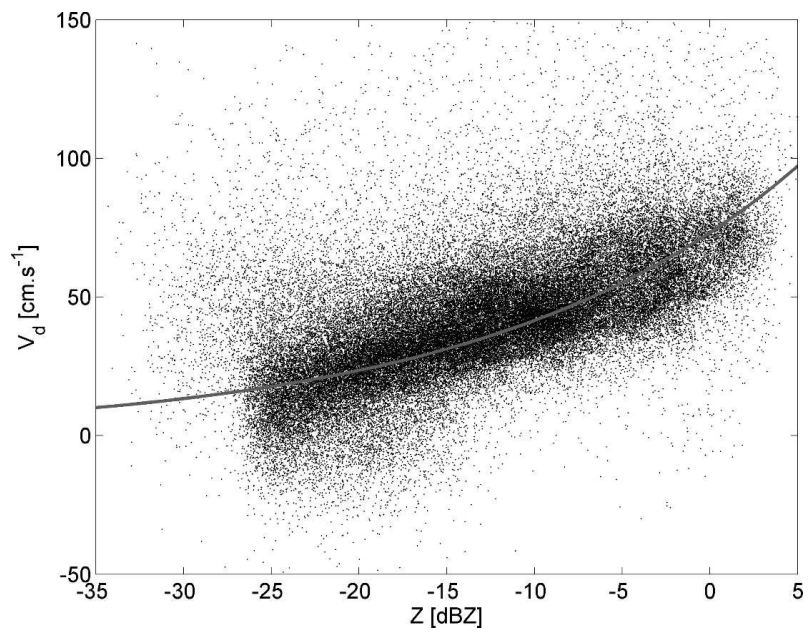

FIG. 3. Time-altitude cross sections of (a) reflectivity $Z$, (b) Doppler velocity $V_{d}$, (c) $V_{t}$ derived from the $V_{t}-Z$ relationship (Fig. 2), and (d) $V_{t}$ derived from the running mean method (RW). These data have been collected by the Radar Aéroporté et Sol de Télédétection des Propriétés Nuageuses (RASTA) 95-GHz cloud radar over the SIRTA, Palaiseau, France, site on 14 Apr 2003.
An alternative approach has been used in the radar method of Matrosov et al. (2002) and has been implemented in RadOn, which consists of estimating terminal fall velocities from 20-min averages of the Doppler velocities. In RadOn this approach has been slightly refined by using 20 -min running means with a 10 -s resolution, which allows for the small-scale variability in the retrievals that is due to possible changes in the cloud microphysical characteristics to be partially kept (referred to as the "running mean" approach in the following). This approach has the great advantage of limiting the assumption of steady microphysics to a $20-\mathrm{min}$ duration horizontally, and to avoid any assumption in the vertical, which seems sensible. The major drawback though is that the vertical air motion will be filtered out in a much less accurate way (occurrences of positive values of fall speed can even be found in the upper part of the clouds, which cannot be treated). The impact of these two approaches on the cloud microphysics retrieval will be analyzed in detail in section 3e, after the method is described.

\section{b. Density and particle habit retrieval from $V_{t}-Z$ relationship}

For different density-diameter relationships and particle habits, we have computed theoretical relationships between the reflectivity-weighted terminal fall velocity and the equivalent reflectivity at 3,35 , and $95 \mathrm{GHz}$, using a very extensive airborne in situ microphysical dataset, including different types of ice clouds, and both midlatitude (CLARE98, CARL99, EUCREX, FASTEX, ARM IOP) and tropical (CEPEX, CRYSTAL-FACE) datasets. This database is described in detail in Delanoë et al. (2005).

In this study, we assume five different area-diameter relationships spanning the most common particle types observed in stratiform clouds. These five area-diameter relationships have been extracted from Mitchell (1996) (solid spheres, hexagonal plates, hexagonal columns, unspherical aggregates, assemblages of planar polycrystals in cirrus clouds). Then for each habit, each $D_{1}$ in the range from 10 to $200 \mu \mathrm{m}$, and each $b_{\rho}$ in the range from -1.4 to -0.5 ), we compute the corresponding $V_{t}-Z$ relationship using the whole microphysical in situ database.

By comparing these theoretical power-law $V_{t}-Z$ relationships with the radar relationship obtained in section $3 \mathrm{a}$, it is therefore possible to determine indirectly the most representative density-diameter and areadiameter relationships for a given cloud. The relationship that produces the smallest difference in the least squares sense with the radar relationship is finally selected. This indirect retrieval is one of the unique features of the method. In other radar or radar-lidar meth- 
ods, these density-diameter and area-diameter relationships are fixed and not adapted from a cloud to another (see, e.g., Hogan et al. 2005; Matrosov et al. 2002; Tinel et al. 2005; Wang and Sassen 2002; Donovan and van Lammeren 2001; Okamoto et al. 2000).

\section{c. $D_{m}$ retrieval from the vertical velocity $V_{t}$}

Once the ice density and area relationships are estimated from the radar and theoretical $V_{t}-Z$ relationships, then all of the relationships of section $2 \mathrm{~b}$ are computed using this ice density. The remaining unknowns used to access the ice cloud properties are $N_{0}^{*}$ and $D_{m}$. To estimate $D_{m}$, we have developed relationships between $V_{t}$ and $D_{m}$ parameterized by the retrieved ice density using the extensive microphysics dataset. The procedure to produce these $V_{t}-\mathrm{D}_{\mathrm{m}}$ relationships is exactly the same as that described for the $V_{t}-Z$ relationships at the end of the previous section.

As shown by Eq. (5), the terminal fall velocity depends only on $D_{m}$ if we assume a normalized PSD shape and a density relationship; $V_{t}$ can then be written as a function of $D_{m}$ as follows:

$$
V_{t}=f\left(D_{m}\right)=g D_{m}^{l},
$$

where $(g, l)$ are related to the retrieved ice density and particle habit.

Then, at this step, only $N_{0}^{*}$ remains to be retrieved in order to access the cloud parameters.

\section{d. $N_{0}^{*}$ from $Z_{e}$ and $D_{m}$}

Regarding $N_{0}^{*}$, there is an analytical relationship between $N_{0}^{*}$ and $D_{m}$ and $Z_{e}$ when an analytical shape is assumed for the normalized PSD,

$$
N_{0}^{*}=\frac{\left|K_{w}\right|^{2} \pi^{5} 10^{-18}}{\lambda^{4}} Z_{e} I\left(D_{m}\right)^{-1}\left(\mathrm{~m}^{-4}\right),
$$

where $Z_{e}$ is in millimeters to the sixth power per cubic meter and $D_{m}$ is in meters, and $I\left(D_{m}\right)$ is an integral function that depends on the ice particle density and the mean volume-weighted diameter,

$$
I\left(D_{m}\right)=\int F\left(D / D_{m}\right) \sigma_{\mathrm{bsc}} d D .
$$

Using the retrieved $N_{0}^{*}$ and $D_{m}$, and $F\left(D / D_{m}\right)$ the normalized PSD, the cloud properties can be finally retrieved from Eq. (8) for IWC, Eq. (9) for $\alpha$, and Eq. (10) for $r_{e}$.

\section{e. Illustration of ice cloud retrieval using RadOn}

The method described in section 3 has been applied to continuous Doppler cloud radar measurements at 95
$\mathrm{GHz}$ collected in the frame of the European Cloudnet project over the Site Instrumental de Recherche par Télédétection Atmosphérique (SIRTA; in Palaiseau, France). The illustrative case shown here is a thick midlatitude prefrontal ice cloud. A backscatter lidar was also operating at that time, but the optical depth of the ice cloud was such that only few hundred meters of the cloud were penetrated by the lidar until complete extinction. This case is therefore a good illustration of the how the radar method and the radar-lidar method complement each other, as the radar method in this particular case allows the upper part of the ice cloud that cannot be reached by the lidar to be explored.

As discussed in section 3a, we have implemented two methods in RadOn to estimate terminal fall speed from the radar measurements, the so-called $V_{t}-Z$ and running mean approaches, which have different advantages and drawbacks.

This is illustrated in Fig. 4, for which both approaches have been applied on the 14 April 2003 case of Figs. $1-3$. The retrievals using the $V_{t}-Z$ approach shows that overall, effective radii are in the correct range for such thick ice clouds (from 20 to $50 \mu \mathrm{m}$ ), but there is no independent estimate of effective radius available for validation. This figure highlights the pros and cons of both methods - on one hand, there are structures in the Doppler velocity field that are not perfectly correlated with the reflectivity as it is shown by Figs. 3a,b, which suggests that there are local changes in the $V_{t}-Z$ relationship. In this case the running mean approach seems more relevant. However, in the upper part of the cloud large artifacts are observed on IWC and $\alpha$, which are not linked to any structure in the reflectivity or Doppler velocity field. These artifacts are responsible for the large discrepancies observed on the time series of optical depth (Fig. 4, lower right panel).

The reason for this instability of the retrieval is explained in Fig. 5, which displays a scatterplot of $N_{0}^{*}$ versus $D_{m}$. As shown by this figure, a small error in $D_{m}$ for the small $D_{m}$ range (and therefore the small $V_{t}$ range) has a dramatic impact on the estimate of $N_{0}^{*}$, which can vary over several orders of magnitude here. It is therefore believed that the small errors resulting from a less accurate filtering of the vertical air velocities when averaging over $20 \mathrm{~min}$ are responsible for the large artifacts observed in the upper part of the cloud. As a result, the current version of RadOn makes use of the $V_{t}-Z$ approach. Furthermore, as will be shown by the comparisons with the radar-lidar methods in section 4 , this version seems to provide fairly accurate and roughly unbiased retrievals of cloud microphysics. Moreover, in the case of the running mean approach, some positive values of fall speed occur, for which the 


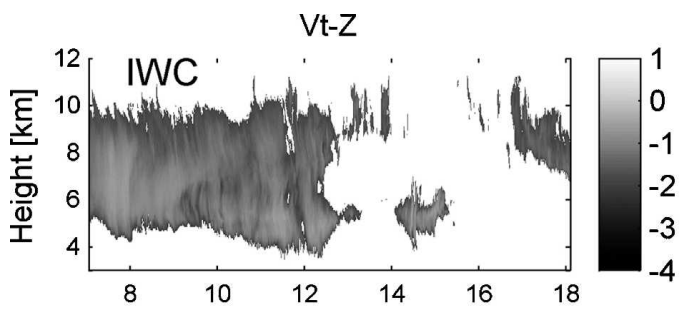

\section{Running mean window}
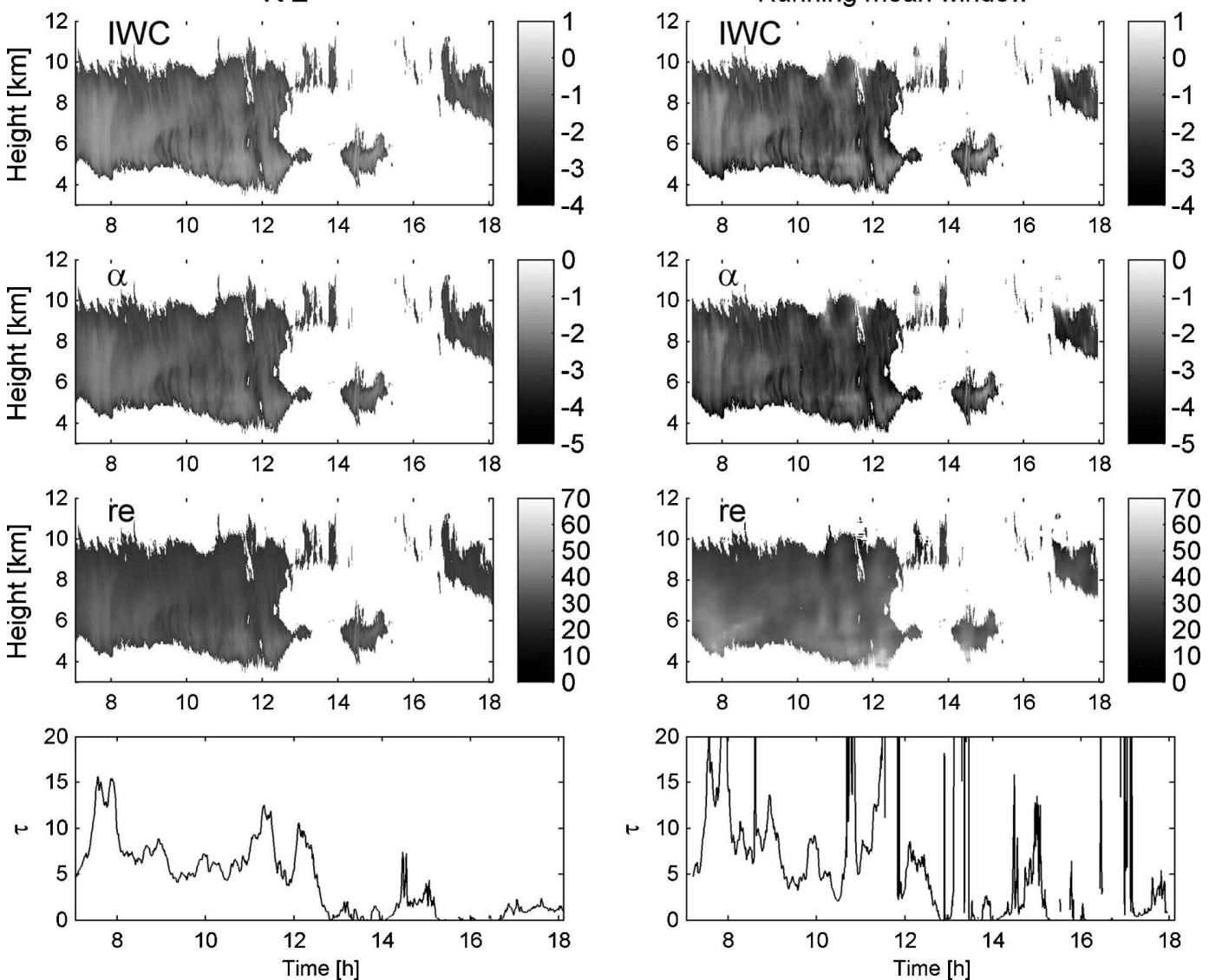

FIG. 4. Time-altitude cross sections of (top to bottom) IWC, $\alpha, r_{e}$, and optical depth as a function of time, (left) determined using $V_{t}$ retrieved from $V_{t}-Z$ relationship and (right) determined using $V_{t}$ from a running mean window. The data were collected by the RASTA $95-\mathrm{GHz}$ cloud radar over the SIRTA, Palaiseau, France, site on 14 Apr 2003.

algorithm is obviously not able to retrieve clouds parameters.

In the next section, a further attempt to evaluate the RadOn method is developed, by quantifying the errors statistics using an extensive aircraft in situ database described earlier, and by comparing the outputs of the RadOn and radar-lidar retrievals in the common sampling area.

\section{Performance of the method}

In this section, rms errors of the RadOn method are characterized, and the different sources of errors are estimated separately in order to evaluate which source of error is most significant. In section $4 a(1)$, we use the microphysics database to assess the global errors, the variability of these errors within the IWC and $\alpha$ ranges, and the variability of the errors when different densitydiameter and area-diameter relationships are assumed.
We then assess the sensitivity of the method to a radar calibration uncertainty, to a residual of vertical air motions in the terminal fall speed, and to both at the same time. We finally come up with an error statistic that is assumed to be reasonably representative of the true errors (end of section 4a). Then, we have estimated and additional sensitivity to the averaging time used for the filtering of the Doppler velocities in order to access terminal fall speed (section $4 \mathrm{~b}$ ). Last, we carry out an intercomparison with a radar-lidar method using $2 \mathrm{yr}$ of continuous radar-lidar observations over three European ground-based sites (section 4c).

\section{a. Error analysis using the microphysics database}

\section{1) GLOBAL RMS ERRORS ON THE CLOUD AND RADIATIVE PARAMETERS WITHOUT INCLUDING THE $V_{t}$ AND DENSITY ERRORS}

In this section, we first develop an error analysis of the method, which does not include the density and $V_{t}$ 


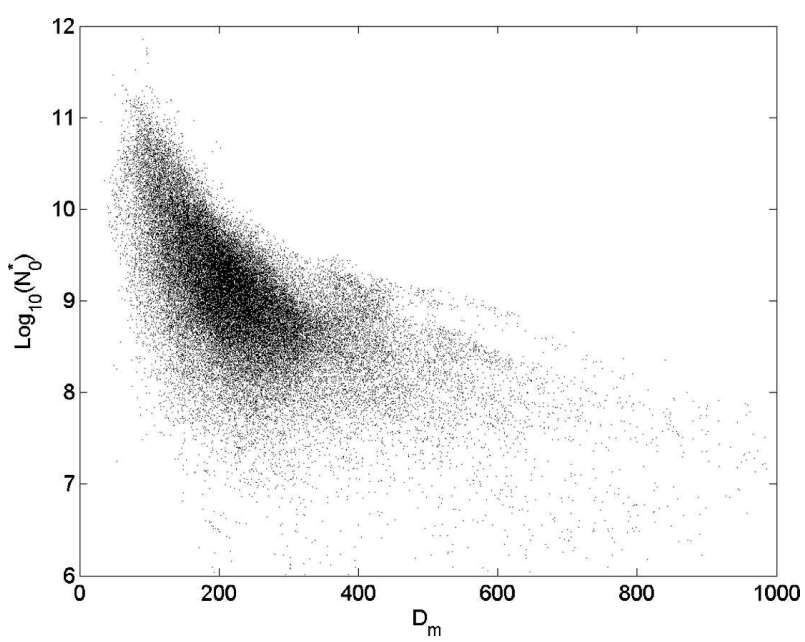

FIG. 5. Logarithm of $N_{0}^{*}$ as a function of $D_{m}$ for an extensive airborne in situ microphysics database.

retrieval errors, and we compare these errors with those obtained in the most recent radar methods using the same error analysis. For this purpose, we compute the reflectivity-weighted velocity and the radar reflectivity at $95 \mathrm{GHz}$ from the extensive microphysics database, assuming the Brown and Francis (1995) densitydiameter relationship $\left(\rho=0.07 D^{-1.1}\right.$, with $D$ in millimeters). These two input parameters $\left(V_{t}\right.$ and $Z$ ) are introduced in RadOn and the outputs are compared with the "true" parameters computed directly from the microphysics database (Fig. 6), using the same densitydiameter relationship as that in RadOn. By doing this, we estimate all sources of errors except the errors associated with the density-diameter relationship and possible errors on $V_{t}$ and $Z$. These other sources of errors are estimated in section $4 \mathrm{a}(2)$. In RadOn, $D_{m}$ is first retrieved from $V_{t}$ and subsequently $N_{0}^{*}$ directly from $Z_{e}$ and $D_{m}$, assuming a density-diameter relationship and an analytical shape for the normalized PSD. We first estimate the errors on these two parameters of the normalized PSD $\left(N_{0}^{*}\right.$ and $\left.D_{m}\right)$. Figure 7 shows the $N_{0}^{*}$ retrieved using the analytical shape (A8) as a function of the true $N_{0}^{*}$ computed directly from the true PSDs for the entire database. The obtained mean relative error and standard deviation on $N_{0}^{*}$, which includes the error resulting from the assumption on the normalized PSD shape, are of about $-2.5 \%$ and $15 \%$, which by construction translates into roughly the same errors on the retrieved cloud parameters (Delanoë et al. 2005). This error is mostly due to the assumption on the normalized PSD shape. Figure 8 shows the $D_{m}$ retrieved from $V_{t}$ using Eq. (12) as a function of the true $D_{m}$ computed directly from the database. As is previously observed for $N_{0}^{*}$, the error is mostly due to the

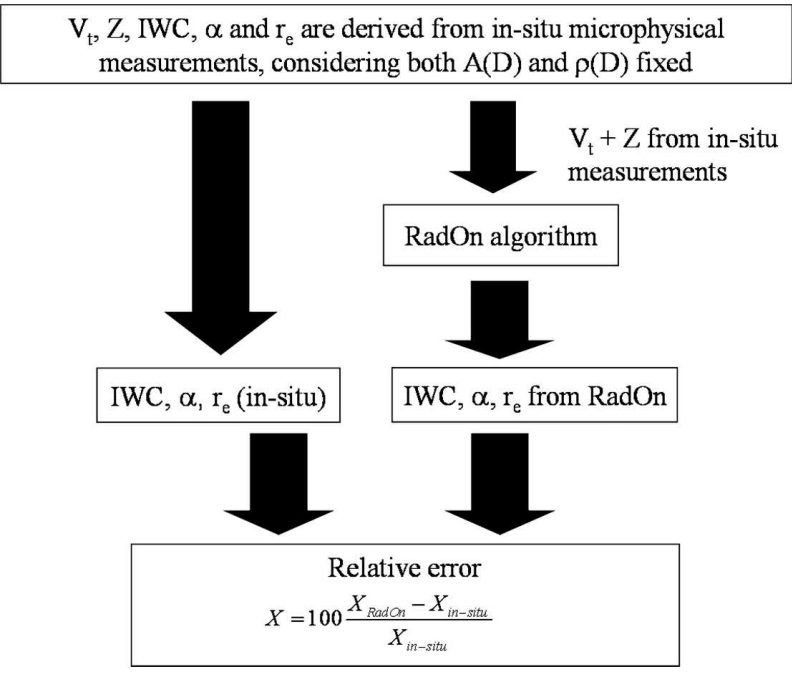

FIG. 6. Evaluation flowchart.

assumption on the normalized PSD shape, a small bias of about $-1.5 \%$ with less than $11 \%$ as standard deviation.

We now turn to the description of the errors on the retrieved cloud properties themselves. The results of this error analysis are summarized in Table 1. All retrieved parameters are in good agreement with the in situ calculations; the bias for the IWC is very small (around $0.4 \%$ ) with a moderate standard deviation [less than $18 \%$, which is much less than the errors of $50 \%-$ $100 \%$ generally acknowledged for IWC-Z/IWC-Z-T relationships with the same sources of errors included; Protat et al. (2007, hereinafter PR07)]. The visible extinction is also in agreement with the in situ calculation, with a bias of about $-3.6 \%$ and a standard deviation of $19 \%$. The retrieved effective radius is slightly overesti-

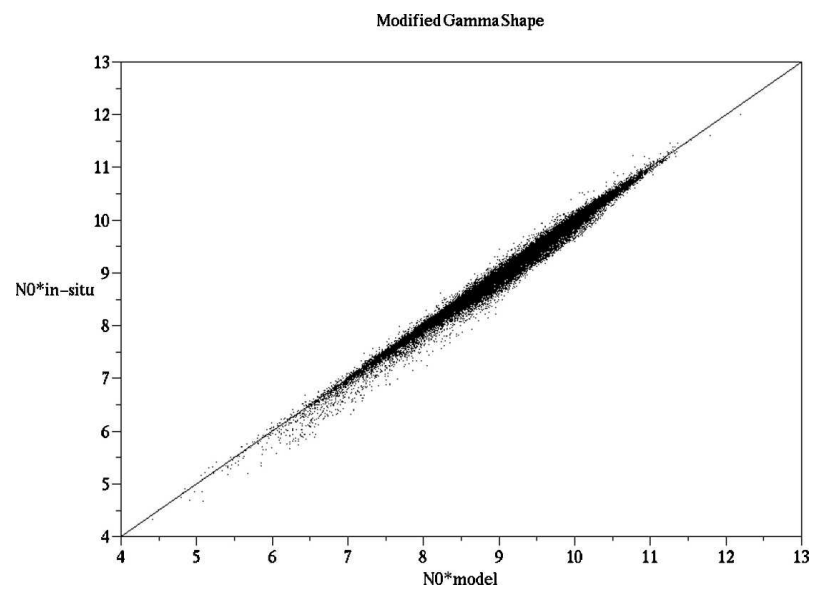

FIG. 7. The $N_{0}^{*}$ retrieved using the analytical shape of Eq. (A8) as a function of the true $N_{0}^{*}$ computed from the true PSDs for an extensive airborne in situ microphysics database. 


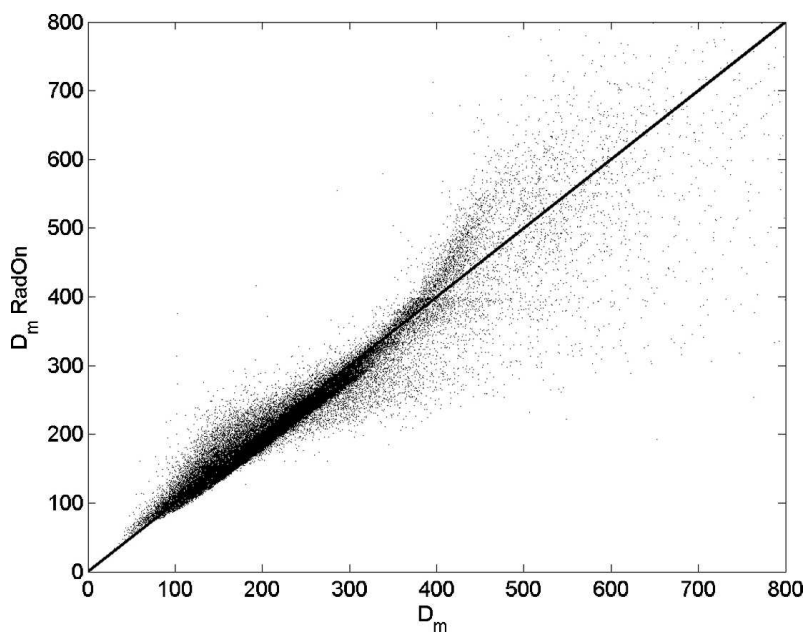

FIG. 8. Scatterplot of $D_{m}$ retrieved from RadOn method (from $\left.V_{t}\right)$ as a function of $D_{m}$ computed from an extensive airborne in situ microphysics database.

mated by the model (5\%), but the standard deviation is less than $11 \%$, which is probably due to the fact that $r_{e}$ depends only on $D_{m}$ and not on $N_{0}^{*}$ [Eq. (10)]. This framework using the microphysics database also offers the opportunity to compare RadOn with other radaronly methods recently published in the literature. We have selected three methods. Two of these, Hogan et al. (2006, hereinafter HO06) and PR07, are statistical relationship methods relating the ice water content to the radar reflectivity and ambient temperature through empirical relationships. IWC- $Z-T$ HO06 relationships are derived from EUCREX and CEPEX datasets, while IWC-Z- $T$ PR07 relationships have been derived from a larger database (CLARE98, CARL99, EUCREX, FASTEX, ARM IOP, CEPEX, and CRYSTALFACE). However, both use the same methodology rigorously. The third one is the Doppler radar method initiated by Matrosov et al. (2002), previously mentioned in the introduction. This latter method uses a $35-\mathrm{GHz}$ radar with a fixed density-diameter relationship, and derives a $D_{0}$ diameter from the terminal fall velocity $V_{t}$ and IWC, and $\alpha$ from $D_{0}$ and $Z$. Results are summarized in Table 1.

For IWC, the standard deviation of RadOn in the Matrosov et al. (2002) Doppler radar methods is better than that of the statistical IWC- $Z-T$ relationships (less than $20 \%$ against more than $58 \%$ ). However, the bias produced by the Matrosov method is higher than IWC$Z-T$ PR07 and RadOn, most likely because of the radar frequency used in this test [Matrosov et al. (2002) is devoted to a $35-\mathrm{GHz}$ radar because it has been built using the Rayleigh approximation] and the fact that the density-diameter relationship is fixed in the Matrosov method while it is adapted for each cloud situation in RadOn. So when particles become larger, the error strongly increases because of the effects of Mie scattering.

For the $\alpha$ retrieval, both Doppler radar methods are very close in terms of standard deviation (15\% for Matrosov and $19 \%$ for RadOn), although the bias of RadOn is much less $(-47 \%$ and $-3.6 \%$, respectively). This larger bias likely results from the Mie effect, as discussed previously.

\section{2) ERror analysis as a Function of $A(D)$ AND $\rho(D)$ FOR A RADAR AT $95 \mathrm{GHz}$}

The purpose of this section is to evaluate whether the errors assuming the Brown and Francis (1995) densitydiameter relationship are similar when other particle habits and density-diameter relationships are considered. Figures 9, 10, and 11 show biases and standard deviations of the relative difference between cloud parameters retrieved from RadOn and those derived from the in situ measurements. Biases and standard deviations are given as a function of five different areadiameter relationships. For each relationship, we change the exponent $b_{\rho}$ of the density-diameter relationships. For this illustration, we fixed the upper limit of the particle diameter to $100 \mu \mathrm{m}\left(D_{1}\right)$. Considering other limit diameters yields similar results (not shown).

As shown in Fig. 9 the bias on the IWC is very small (less than $0.4 \%$ ) and the standard deviation is around $18.1 \%$, assuming $b_{\rho}=-1.1$. If we consider extreme values of the exponent of $\rho(D)$ (from -1.4 to -0.5 ), the errors are not very different. The overestimation of IWC is less than $5.5 \%$ with a standard deviation less than $20 \%$ for $b_{\rho}=-1.4$. IWC is underestimated (around $8 \%$ ) with the same standard deviation (21\%) for $b_{\rho}=-0.5$. The error does not depend on the area-

TABLE 1. Comparison error analysis between RadOn and the other methods.

\begin{tabular}{|c|c|c|c|c|c|c|c|c|}
\hline & \multicolumn{2}{|c|}{ RadOn } & \multicolumn{2}{|c|}{ Matrosov et al. (2002) } & \multicolumn{2}{|c|}{ IWC- $Z-T$ HO06 } & \multicolumn{2}{|c|}{ IWC-Z-T PR07 } \\
\hline & Bias & $\sigma$ & Bias & $\sigma$ & Bias & $\sigma$ & Bias & $\sigma$ \\
\hline IWC & 0.4 & 17.3 & -28 & 18 & 43.5 & 76.7 & 24.0 & 58.6 \\
\hline$\alpha$ & -3.6 & 18.7 & -47 & 15 & - & - & - & - \\
\hline$r_{e}$ & 5.2 & 10.5 & 40 & 22 & - & - & - & - \\
\hline
\end{tabular}




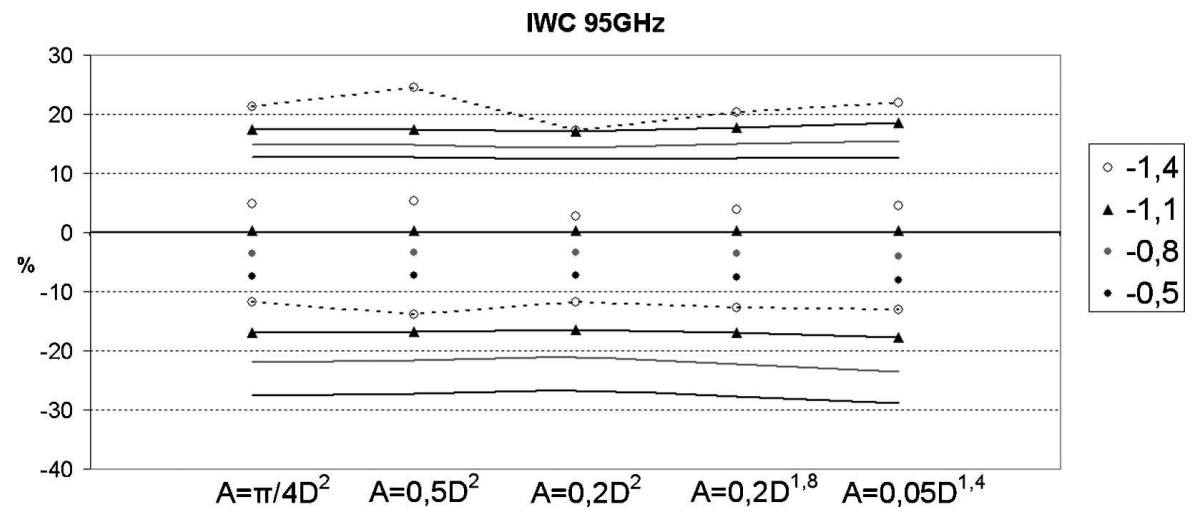

FIG. 9. Bias (points by themselves) and standard deviation (plotted lines) of the relative error in IWCs retrieved from RadOn (95-GHz radar) and those derived from in situ measurements as a function of the five different area-diameter relationships listed at the bottom of the figure. For each relationship, the exponent of the density-diameter relationship is changed to the values listed on the right side of the figure.

diameter relationship when $b_{\rho}$ varies between -0.5 and -1.1 .

Figure 12 shows the bias and standard deviation as a function of $b_{\rho}$ for IWC. The bias is positive when $b_{\rho}<$ -1.1 and negative above -1.1 . The standard deviation increases slowly with $b_{\rho}$, from $15 \%$ to $21 \%$. Whatever the density-diameter relationship, as is shown by Fig. 10 , we underestimate $\alpha$ from $-2 \%$ to $-10 \%$. Contrary to the case of IWC, the standard deviation of the relative difference in $\alpha$ is strongly dependent on the areadiameter relationship. It increases strongly as $b_{\rho}$ decreases and the particle type departs strongly from the sphere (Fig. 13). The largest error (bias $=-10 \%$ and std $=37.8 \%)$ is obtained when the area-diameter relationship is $A(D)=0.05 D^{1.4}$, which corresponds to the hexagonal columns for the particle larger than $300 \mu \mathrm{m}$ (Mitchell 1996). However, in the literature this areadiameter relationship is encountered only when $b_{\rho}$ is less than -1 (Mitchell 1996). When the area-diameter relationship is closer to the spherical particle (or plates) the bias is around $4 \%$ and the standard deviation is $28 \%$. Globally, biases and standard deviations increase with $b_{\rho}$.

The effective radius is proportional to the ratio IWC $/ \alpha$ (Francis et al. 1994). As shown in Fig. 11, $r_{e}$ is slightly overestimated (from $4 \%$ to $16 \%$ ). As for $\alpha$, biases and standard deviations are larger for the combination $A(D)=0.05 D^{1.4}$ and $b_{\rho}=-0.5$. However, if we remove this unlikely configuration (discussed previously), the bias does not exceed $10 \%$ and the standard deviation is less than $16 \%$. It is noteworthy that the same study conducted with a radar frequency of 35 $\mathrm{GHz}$ yielded similar results (not shown).

\section{3) ERRor VARIABILITY AS A FUNCTION OF IWC AND $\alpha$}

In this section, we estimate the relative root-meansquare difference (rmsd) between cloud parameter

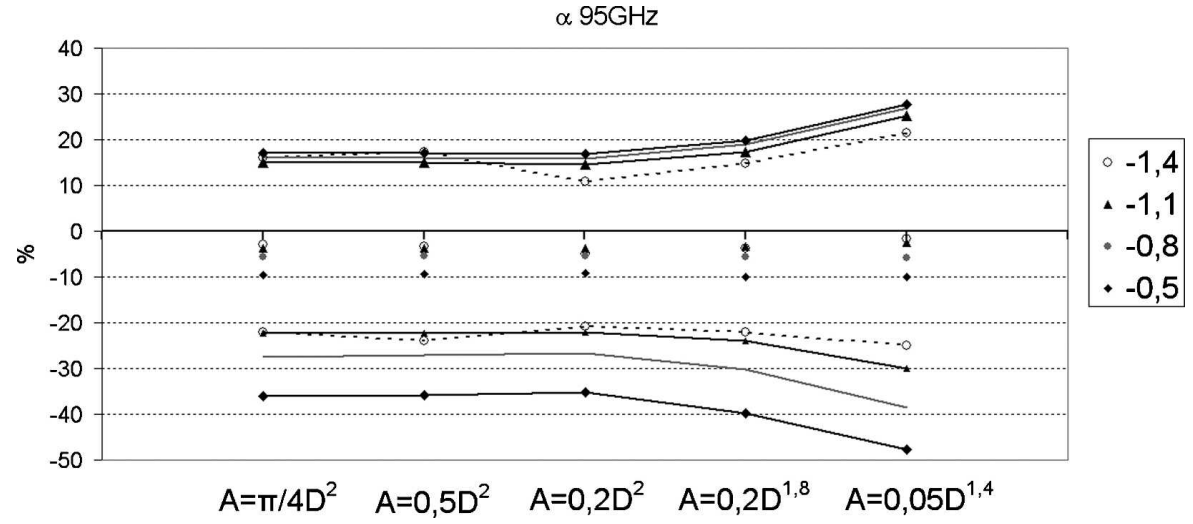

FIG. 10. Same as Fig. 9, but for visible extinction $\alpha$. 




FIG. 11. Same as Fig. 9, but for effective radius $r_{e}$.

(IWC, $\alpha$ ) computed directly from the measured PSDs and the RadOn retrieval of these parameters as a function of the logarithm of the evaluated parameter. The rmsd characterizes both the bias and the standard deviation of the relative difference. In this section, we focus solely on the ice water content and the visible extinction, because the effective radius is deduced from the ratio IWC/ $\alpha$ and its rmsd is less than $15 \%$ (not shown), whatever the area-diameter relationship considered. This study has been conducted using several combinations of $A(D)$ and $\rho(D)$ relationships, as previously, but we only show the result for the Brown and Francis (1995) relationship.

Figure 14a shows the relative rmsd on IWC as a func- tion of $\log (\mathrm{IWC})$ and confirms the previous results; the error does not depend on the choice of $A(D)$. The rmsd is nearly constant (about 20\%) through the $\log$ (IWC) range. However, as shown in Fig. 14b, $\alpha$ is a little bit more dependent on $A(D)$. The rmsd decreases slightly when $\log (\alpha)$ increases in the range from $10^{-6}$ to $10^{-3}$ and is $10 \%$ greater for the hexagonal columns than for the other $A(D)$ (as seen in the previous section).

\section{4) RAdOn SENSITIVITY TO A CALIBRATION ERROR AND TO $V_{t}$ ERRORS}

Unfortunately, the radar measurements are not perfect and an error on the $V_{t}$ retrieval from the $V_{t}-Z$ relationship can reach from \pm 5 to $\pm 10 \mathrm{~cm} \mathrm{~s}^{-1}$ (Protat

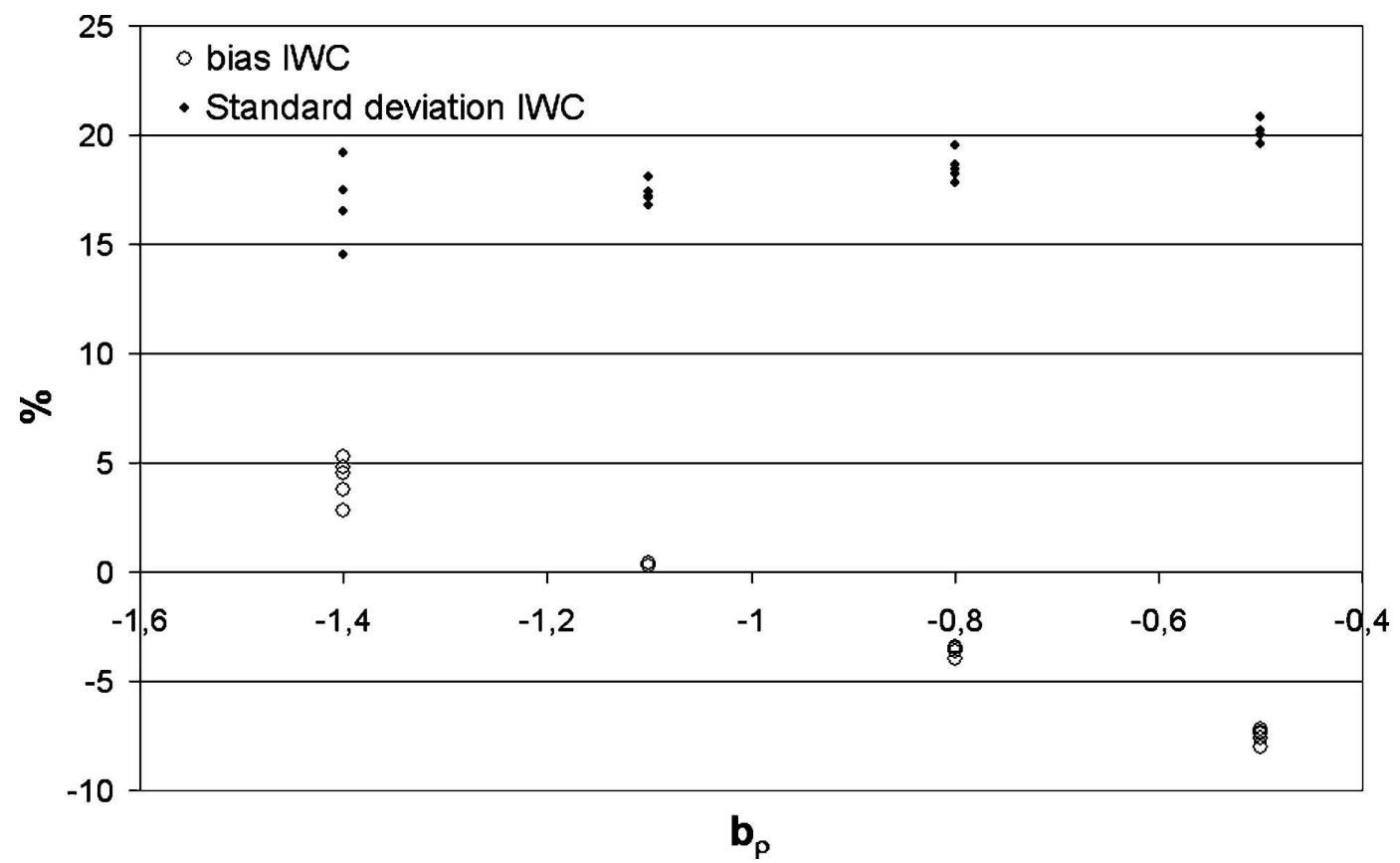

FIG. 12. Bias and standard deviation of the relative error in IWC retrievals with the area-diameter relationships shown in Fig. 9 combined as a function of the four density exponent $b_{\rho}$ s listed in Fig. 9. 


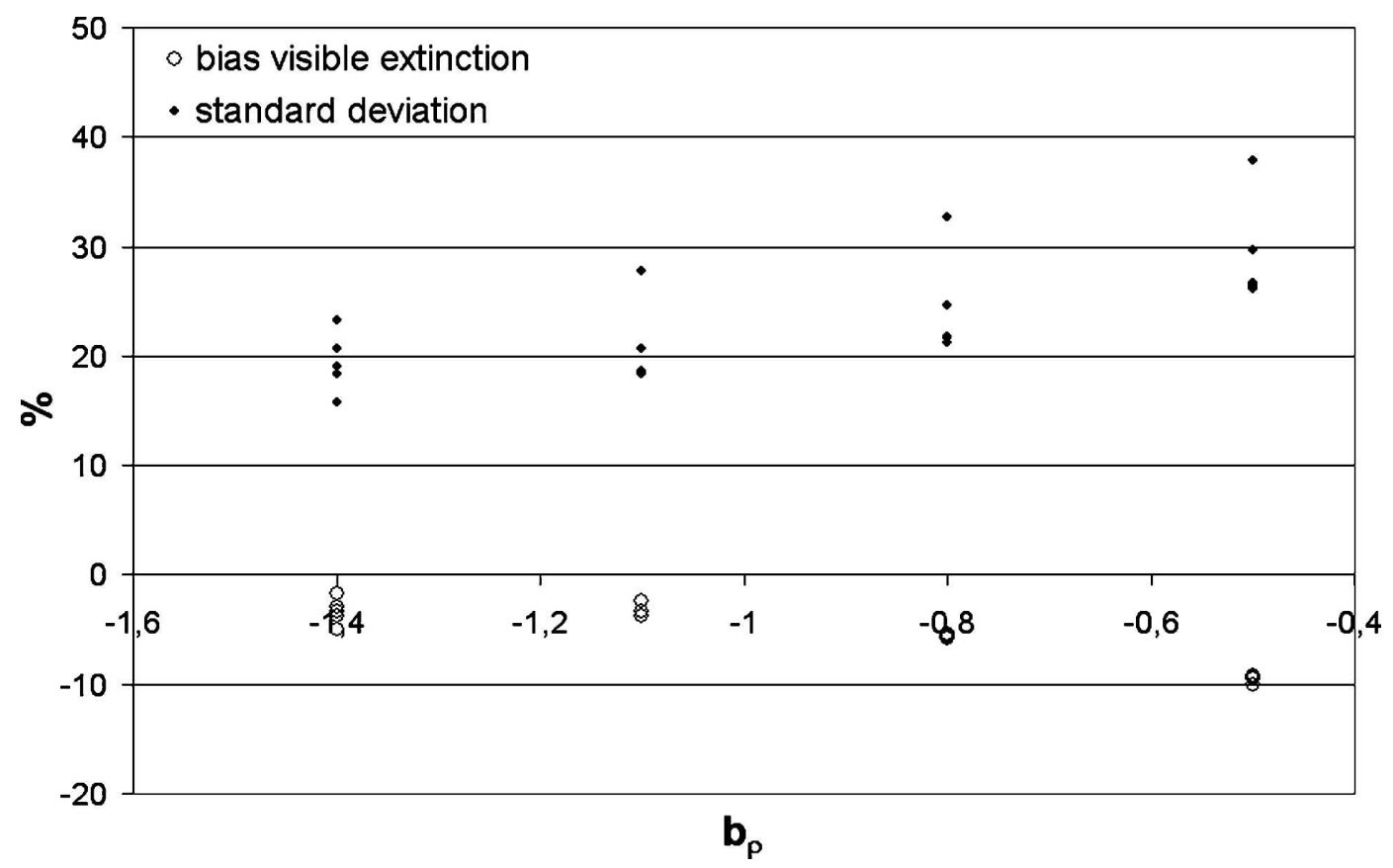

FIG. 13. As in Fig. 12, but for visible extinction $\alpha$.

et al. 2003), which roughly corresponds to the synoptic environmental uplift in a cloud. Potential effects of a change in the microphysical characteristics or an increase of the $V_{t}$ error resulting from the time lag used to filter out the small-scale vertical air motions can be treated as sources that increase the $V_{t}$ variability for a given $Z$. This is why we have also estimated the errors arising from an addition of a Gaussian noise of a given standard deviation to $V_{t}$. In addition, although cloud radars can be fairly accurately calibrated, it is difficult to achieve accuracies better than $1 \mathrm{~dB}$, which needs to be accounted for in our error calculations. It is also important to note that these two errors indirectly produce errors in the retrieval of the density-diameter relationship. As a result, this last sensitivity test can be viewed as a representative estimate of the total true errors of RadOn.

In this section we first estimate the sensitivity of RadOn to a calibration error on radar reflectivity $(+1$ and then $+2 \mathrm{dBZ})$ and to a random noise on terminal fall velocity $\left( \pm 5, \pm 10 \mathrm{~cm} \mathrm{~s}^{-1}\right)$, in addition to the other sources of errors estimated previously. As is shown in Table 2, $\mathrm{a}+1(+2) \mathrm{dBZ}$ calibration error translates into a bias on IWC and $\alpha$ of about $18 \%$ and $25 \%$ (35\% and $40 \%$ ), which is larger than previously estimated in Table 1. However, the standard deviation of the errors on the clouds parameters is less sensitive (e.g., $+1 \mathrm{dBZ}$ leads to $\sigma_{\text {IWC }}=13.4$, as compared with $18 \%$ in Table $1)$. Moreover, there is only the effect on the PSD shape and $D_{m}$ retrieval on the effective radius.
When we apply a Gaussian noise with a $\pm 5 \mathrm{~cm} \mathrm{~s}^{-1}$ standard deviation on $V_{t}$, the biases on IWC and $\alpha$ do not increase much (less than around 5\%) and the standard deviation is around $32 \%$. If we increase the standard deviation of the Gaussian noise up to $\pm 10 \mathrm{~cm} \mathrm{~s}^{-1}$, the bias reaches $22.5 \%$ and the standard deviation is $70 \%-80 \%$. Again, the effective radius is not sensitive to the $V_{t}-Z$ random error, the bias is only due to the PSD assumption, and the $D_{m}$ retrieval error and standard deviation is less than $12 \%$.

As discussed previously it is expected that there should be a residual vertical air motion after filtering due to the synoptic uplift. Therefore, we now investigate the error of the method if the terminal fall velocity is shifted with a $+10 \mathrm{~cm} \mathrm{~s}^{-1}$ vertical air motion contribution. The determination of the density-diameter and area-diameter relationships is also affected by the error on $V_{t}$. Figure 15 represents the relative rms difference between the true IWC and $\alpha$ [from the in situ measurements when we apply the Brown and Francis (1995) relationship] and IWC and $\alpha$ obtained by adding up to $+10 \mathrm{~cm} \mathrm{~s}^{-1}$ to $V_{t}$. The errors are shown as a function of the true IWC and $\alpha$. In this case the error in IWC does not exceed $30 \%$ and remains less than $20 \%$ for the IWC range of $2 \times 10^{-3}$ to $0.1 \mathrm{~g} \mathrm{~cm}^{-3}$. The increase of the error owing to this bias is unexpectedly small, and even smaller than without the bias in the small IWC bins. The effect of this bias is to increase the error for the large IWCs, but surprisingly to reduce the errors for the small IWCs. This is most likely the result of a different 

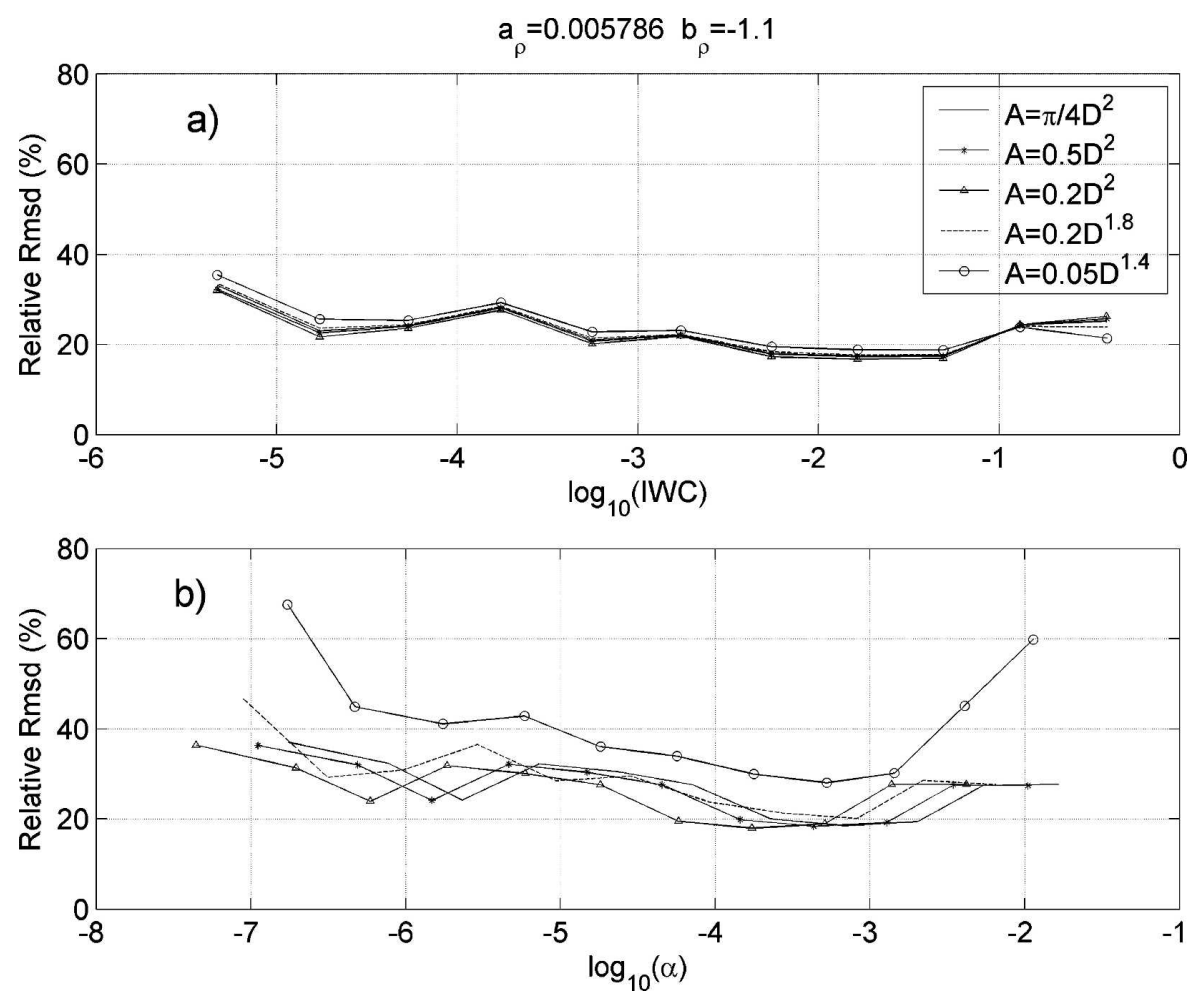

FIG. 14. Relative rms difference in (a) IWC as a function of the $\log (\mathrm{IWC})$ and (b) $\alpha$ as a function of $\log (\alpha)$ for a $95-\mathrm{GHz}$ radar and the area-diameter relationships listed.

adjustment of the density-diameter and area-diameter relationships in the retrieval. Regarding $\alpha$, the effect of a $10 \mathrm{~cm} \mathrm{~s}^{-1}$ bias on $V_{t}$ is obviously larger than that for IWC, with errors of around $40 \%-50 \%$, corresponding to an increase by around $30 \%$ over the whole a range with respect to the case of perfect $V_{t} \mathrm{~s}$.

When a $1-\mathrm{dB} Z$ calibration error is introduced in $Z$, the error statistics on IWC is not modified with respect to the case of a perfect calibration for IWC less than $10^{-1.5} \mathrm{~g} \mathrm{~m}^{-3}$ (the error is less than $20 \%$ ), while it increases up to $30 \%$ for IWC larger than $10^{-1.5} \mathrm{~g} \mathrm{~cm}^{-3}$ (a $10 \%$ increase with respect to the case of a perfect calibration). The increase of the error on $\alpha$ is around $10 \%$ over the whole range, yielding relative errors on $\alpha$ of

TABLE 2. Sensitivity of RadOn retrievals to calibration and $V_{t}-Z$ errors.

\begin{tabular}{|c|c|c|c|c|c|c|}
\hline & \multicolumn{2}{|c|}{ IWC } & \multicolumn{2}{|c|}{$\alpha$} & \multicolumn{2}{|c|}{$r_{e}$} \\
\hline & Bias & $\sigma$ & Bias & $\sigma$ & Bias & $\sigma$ \\
\hline$+1 \mathrm{~dB} Z$ & 17.4 & 13.4 & 24.3 & 13.7 & 5.2 & 10.5 \\
\hline$+2 \mathrm{~dB} Z$ & 34.4 & 10.6 & 39.8 & 10.8 & 5.1 & 10.5 \\
\hline$V_{t}: \pm 5 \mathrm{~cm} \mathrm{~s}^{-1}$ & 5.3 & 32.5 & 1 & 32 & 5.1 & 10.7 \\
\hline$V_{t}: \pm 10 \mathrm{~cm} \mathrm{~s}^{-1}$ & 22.5 & 81.6 & 16.3 & 70 & 5.2 & 11.5 \\
\hline
\end{tabular}

around $30 \%$. It is noteworthy that the effect on $\alpha$ of a calibration error is much less than the effect of a bias on $V_{t}$.

To estimate an error statistic that is as realistic as possible for RadOn, we finally added both $10 \mathrm{~cm} \mathrm{~s}^{-1}$ to $V_{t}$ and $1 \mathrm{dBZ}$ to $Z$. This translates into an error on IWC of less than $30 \%$ for IWC less than $0.06 \mathrm{~g} \mathrm{~m}^{-3}$, which increases up to $40 \%$ for the larger IWCs. However, IWC is not really sensitive to a calibration error or a $V_{t}$ bias if we treat them separately, except for the large IWCs. The error on the extinction is around $55 \%-60 \%$ over the whole range of extinctions. Errors resulting from $V_{t}$ and calibration are additive in the case of extinction, which is not the case for IWC, except for large IWCs.

\section{b. Sensitivity of the retrieval to the Doppler velocity averaging period}

Using the 14 April 2003 case (previously described in section $3 \mathrm{e}$ ), we have investigated the impact of the time lag used to filter out the vertical air motions in the Doppler velocities on the retrieval. To do so, we have derived $V_{t}-Z$ relationships every 1,2 , and $3 \mathrm{~h}$, and then we have compared the results with the $V_{t}-Z$ derived 

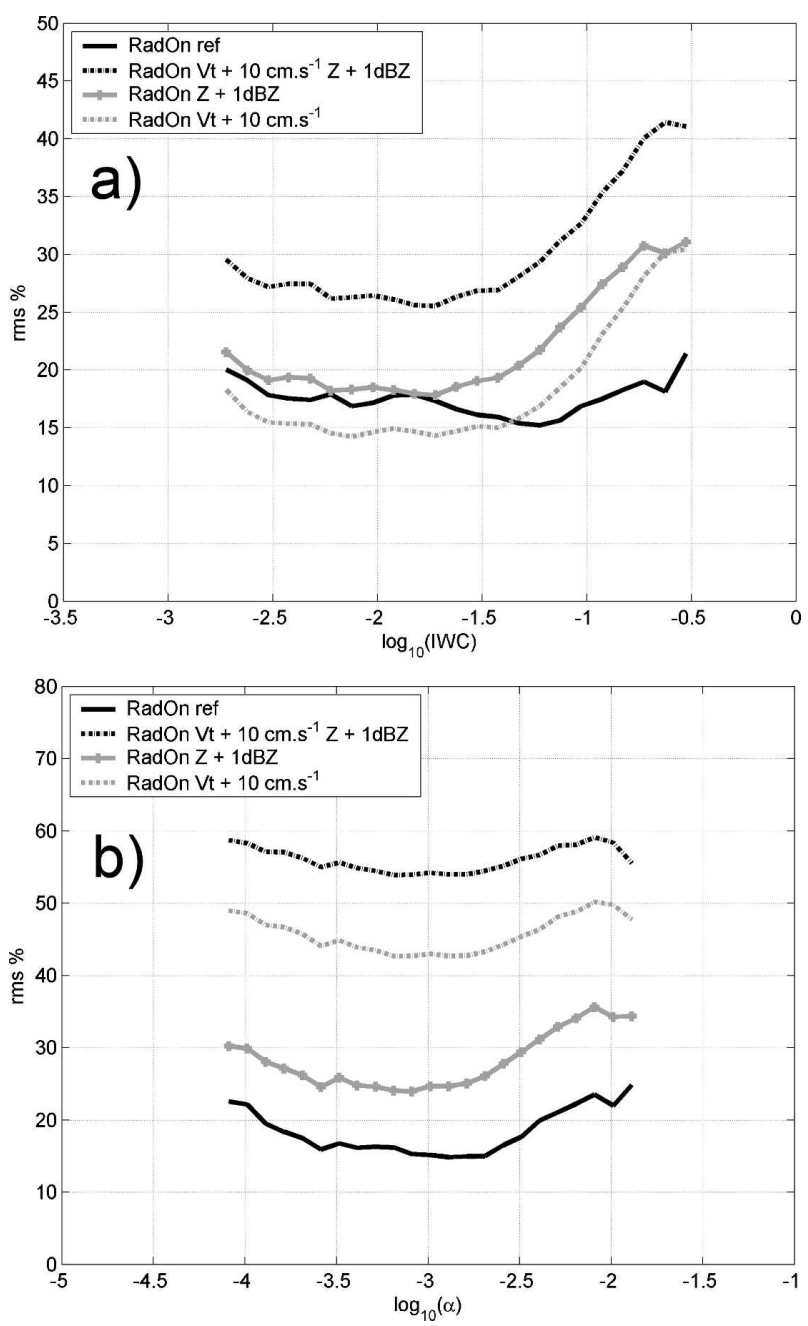

FIG. 15. Relative rms difference in (a) IWC as a function of the $\log ($ IWC) and (b) $\alpha$ as a function of $\log (\alpha)$ for a $95-\mathrm{GHz}$ radar. Solid black lines represent RadOn results with the Brown and Francis density-diameter relationship and unbiased $V_{t}$ and $Z$. Solid gray lines with circles represent the results for a $Z$ bias of 1 dBZ. Dashed gray lines correspond to a $V_{t}$ bias of $10 \mathrm{~cm} \mathrm{~s}^{-1}$. Dashed black lines correspond to the bias in both $V_{t}$ and $Z$. Bins filled with less than 500 points are not displayed.

using the whole time period (approximately $17 \mathrm{~h}$ for this particular case). The results have been summarized in Table 3 for $V_{t}, \alpha$, and IWC. As suggested by Table 3, the effect of the averaging period on $V_{t}$ and $\alpha$ is essentially to increase the standard deviation of the error from $8 \%$ for a 3 -h averaging up to $16 \%$ for a 1 -h averaging for $V_{t}$, and from $20 \%$ for a 3-h averaging up to $56 \%$ for a 1 -h averaging for $\alpha$. Regarding IWC, the averaging tends to produce a bias (which was not the case for $V_{t}$ and $\alpha$ ) increasing from $-9 \%$ for $3 \mathrm{~h}$ to $-22 \%$ for a $1-\mathrm{h}$ averaging. Conversely, the increase in standard deviation $(11 \%$, less than in Table 1 , up to
TABLE 3. Sensitivity of the retrieval to the Doppler velocity averaging period.

\begin{tabular}{|c|c|c|c|c|c|c|}
\hline & \multicolumn{2}{|c|}{$1 \mathrm{~h}$} & \multicolumn{2}{|c|}{$2 \mathrm{~h}$} & \multicolumn{2}{|c|}{$3 \mathrm{~h}$} \\
\hline & Bias (\%) & $\sigma(\%)$ & Bias (\%) & $\sigma(\%)$ & Bias (\%) & $\sigma(\%)$ \\
\hline$V_{t}$ & -0.7 & 16. & -0.2 & 9.8 & -1.2 & 8.1 \\
\hline IWC & -22.4 & 24.3 & -9.6 & 14.8 & -9.6 & 11.2 \\
\hline$\alpha$ & -6.6 & 56.1 & -2.7 & 29.7 & 1.6 & 19.6 \\
\hline
\end{tabular}

$24 \%$ for 1-h averaging) is smaller than that observed for $V_{t}$ and $\alpha$.

As it appears from Table 3-that using a 1-h averaging period tends to degrade the quality of the retrieval-we suggest that the averaging period should not be less than $2 \mathrm{~h}$.

\section{c. Comparison with radar-lidar method}

To evaluate the performance of RadOn statistically, it is compared in the present section with the radarlidar retrieval method of Tinel et al. (2005) in the cloud areas sampled by both the radar and the lidar. This is done using the whole Cloudnet radar-lidar database (Illingworth et al. 2007) collected during 2 yr from three European instrumented sites (Chilbolton, United Kingdom, Cabauw, Netherlands, and Palaiseau, France).

From Figs. 16-17, it appears clearly that the RadOn and radar-lidar methods are statistically consistent, with a roughly unbiased estimate of IWC and $\alpha$ over the whole IWC/ $\alpha$ variability range.

The standard deviation is also not very large (22\%), as shown by the fact that most points are close to the $1: 1$

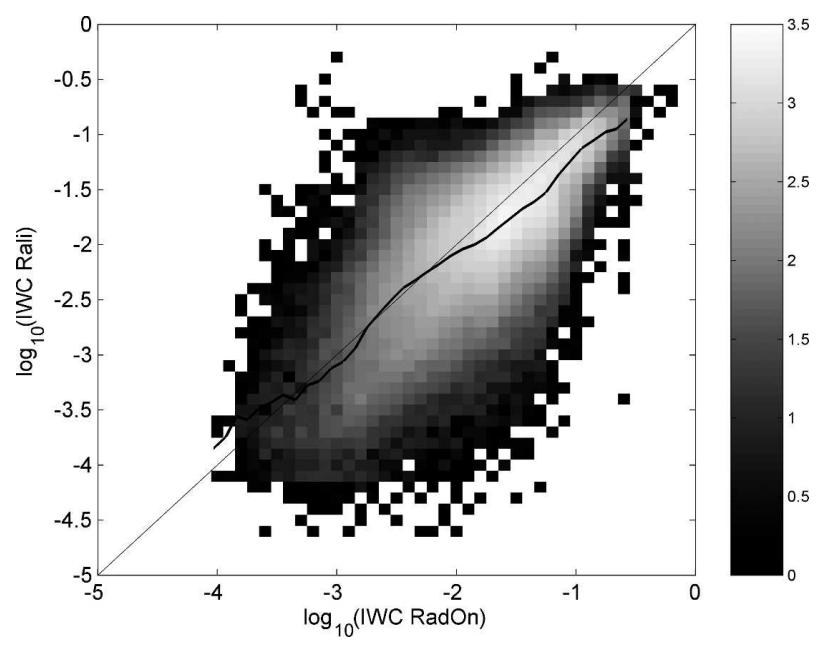

FIG. 16. $\log$ (IWC) derived from radar-lidar (Rali) measurements as a function of $\log$ (IWC) derived from RadOn. Shading represents the logarithm of density points (with black representing 0 and gray to white representing $4 \rightarrow 10^{3.5}$ points). The black line corresponds to the mean difference. 


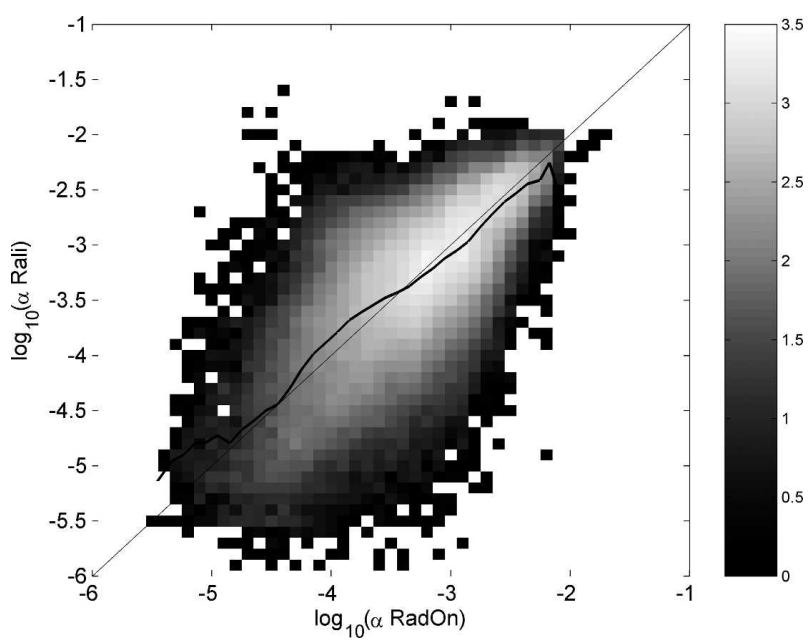

FIG. 17. As in Fig. 16, but for visible extinction $\alpha$.

line (gray to white dots). It is however observed that for intermediate to large IWCs $\left(>10^{-2} \mathrm{~g} \mathrm{~m}^{-3}\right)$ a bias appears, with IWCs retrieved with RadOn being larger than those retrieved with the radar-lidar method. This is due to the Mie effect (Mie 1908), which is not accounted for in the radar-lidar method, while it is in RadOn; thus, this is more likely to be a problem of the radar-lidar method.

It is particularly impressive to see in Fig. 17 how the estimate of extinction by the radar is good when compared with the much more direct estimate by the radarlidar method. This is despite the fact that extinction is only very indirectly derived in RadOn from the $A(D)$ relationship selected in the step described in section $3 \mathrm{~b}$, whereas it is directly proportional to the backscatter measured by the lidar. This good agreement indirectly validates the retrieval of density-diameter and areadiameter relationships. Furthermore, as mentioned by Hogan et al. (2005), the visible extinction retrieved from the radar-lidar algorithm is not sensitive to the $A(D)$ and $\rho(D)$ assumptions.

\section{Conclusions}

A new method for retrieval of ice cloud properties from ground-based Doppler radar observations (called RadOn) has been described in the present paper. From the Doppler velocity and radar reflectivities we retrieve IWC, $\alpha, r_{e}$, and the optical thickness using the normalized PSD concept $\left[N(\mathrm{D})=N_{0}^{*} F\left(D / D_{m}\right)\right]$. The terminal fall velocity is derived from the Doppler velocity using a $V_{t}-Z$ relationship. From this relationship, we estimate the most representative density-diameter and areadiameter relationships, by comparing the radar $V_{t}-Z$ with in situ $V_{t}-Z$ relationships. The relationship that produces the smallest difference in the least squares sense with the radar relationship is selected. Once the density- and area-diameter relationships are fixed, we derive $D_{m}$ from $V_{t}$ and $N_{0}^{*}$ from $D_{m}$ and $Z$.

This method has then been evaluated using an extensive microphysics in situ database. We have first carried out an error analysis assuming a perfect measurement of the terminal fall velocity and the radar reflectivity and no error in the density-diameter relationship retrieval. All retrieved parameters are in good agreement with the in situ calculations; the bias for the IWC is very small (around $0.4 \%$ ), with a moderate standard deviation (less than 18\%). The visible extinction is also in agreement with the in situ calculation, with a bias of about $-3.6 \%$ and a standard deviation very close to $19 \%$. The retrieved effective radius is overestimated by the model (5\%), but the standard deviation is less than $11 \%$. Moreover, this analysis has been conducted with several density-diameter and area-diameter relationships, yielding similar results.

We then carried out a new error evaluation, taking into account a radar calibration error and a residual in the terminal fall velocity. The impact of a radar calibration error, when we assume no error on the density retrieval, is to produce a bias of $20 \%-25 \%$ in the cloud parameters (for 1-dBZ error), while a random $V_{t}$ error tends to increase the standard deviation from $18 \%$ to $32 \%$ (for a $\pm 5 \mathrm{~cm} \mathrm{~s}^{-1}$ random noise). Because the two effects are very similar on IWC and $\alpha$, the effective radius is not really affected. When we take into account both density retrieval errors-the potential radar calibration error and a $10 \mathrm{~cm} \mathrm{~s}^{-1}$ bias in $V_{t}$-the final relative rms error in IWC and $\alpha$ are $30 \%-40 \%$ and $55 \%-$ $60 \%$, respectively.

We also compared the RadOn retrievals with coincident radar-lidar retrievals and showed that the estimate of extinction by radar is good when compared with the much more direct estimate by the radar-lidar method. This is despite the fact that extinction is only very indirectly derived in RadOn from the $A(D)$ relationship selected, whereas it is directly proportional to the backscatter measured by the lidar. This good agreement indirectly validates the retrieval of densitydiameter and area-diameter relationships. We also presently participate to an intercomparison exercise using measured in situ IWC profiles and PSDs, from which radar reflectivities and Doppler fall speeds are simulated. This should be compared with the present error estimates when available.

This method is presently being systematically applied to the cloud radar measurements collected over the three instrumented sites of the European Cloudnet project to evaluate the representation of ice clouds in 
numerical weather prediction models and to build up a cloud climatology.

Acknowledgments. This work was carried out with the support of EU Cloudnet Contract EVK2-CT-200000064, and the funding from the Centre National d'Etudes Spatiales (CNES) and Institut National des Sciences de l'Univers (INSU) in the frame of the RALI project. The authors are also grateful to Drs. R. J. Hogan and E. J. O'Connor for providing the categorization and classification Cloudnet files that made the algorithm application to the Cloudnet database easier.

\section{APPENDIX}

\section{Recall of the Principle of the Normalization of the PSD and Main Results}

In this appendix, we summarize the important results of the normalized particle size distribution study of Delanoë et al. (2005), and we encourage the reader to refer to the original paper for more details.

The convenient formulation of the "equivalent melted diameter" has been chosen for use instead of the physical diameter, which corresponds to the diameter the ice particle would have if it was a spherical water particle of the same mass. This formulation implies that a density-diameter relationship must be assumed. By definition, the particle size distribution $N\left(D_{\text {eq }}\right)$ is the number of particles per unit volume and per interval of diameter $\left(\mathrm{m}^{-4}\right)$, where $D_{\text {eq }}$ is the melted equivalent diameter $(\mathrm{m})$. This formalism, known as the "normalized PSD," consists of scaling the diameter and $N\left(D_{\text {eq }}\right)$ axes in such a way that the PSDs are independent of the ice water content (IWC) and the mean volume-weighted diameter $\left(D_{m}\right)$.

Let us recall that IWC $\left(\mathrm{g} \mathrm{m}^{-3}\right)$ is proportional to the third moment of the PSD and $D_{m}(\mathrm{~m})$ is proportional to the ratio of the fourth to the third moment of the PSD:

$$
\begin{aligned}
D_{m} & =\frac{\int N\left(D_{\mathrm{eq}}\right) D_{\mathrm{eq}}^{4} d D_{\mathrm{eq}}}{\int N\left(D_{\mathrm{eq}}\right) D_{\mathrm{eq}}^{3} d D_{\mathrm{eq}}} \text { and } \\
\mathrm{IWC} & =\frac{\pi \rho_{w}}{6} \int N\left(D_{\mathrm{eq}}\right) D_{\mathrm{eq}}^{3} d D_{\mathrm{eq}} .
\end{aligned}
$$

A general expression of the PSD can be written as

$$
N\left(D_{\mathrm{eq}}\right)=N_{0}^{*} F\left(D_{\mathrm{eq}} / D_{m}\right)
$$

where $N_{0}^{*}$ is the scaling parameter for the concentration axis, $D_{m}$ is the scaling parameter for the diameter axis, and $F$ denotes the normalized PSD.
Assuming (A1), then by definition $F(X)$ satisfies the following equation:

$$
\int_{0}^{\infty} F(X) X^{4} d X=\int_{0}^{\infty} F(X) X^{3} d X .
$$

Then, considering (A2), we can also write

$$
\int_{0}^{\infty} F(X) X^{3} d X=\frac{6}{\pi \rho_{w}} \frac{\mathrm{IWC}}{N_{0}^{*} D_{m}^{4}} .
$$

It follows from (A4) and (A5) that to make the normalized PSD independent of IWC and $D_{m}$, the third moment of the PSD must be constant. This constant has been chosen in such a way that the $N_{0}^{*}$ parameter is equal to the intercept parameter $N_{0}$ of the exponential Marshall and Palmer (1948) PSD, which yields

$$
\int_{0}^{\infty} F(X) X^{3} d X=C=\frac{\Gamma(4)}{4^{4}} .
$$

The $N_{0}^{*}$ parameter is a function of IWC and $D_{m}$, which can be written as

$$
N_{0}^{*}=\frac{4^{4}}{\pi \rho_{w}} \frac{\mathrm{IWC}}{D_{m}^{4}}\left(\mathrm{~m}^{-4}\right) .
$$

We have investigated the statistical properties of the normalized particle size distribution in ice clouds. To do so, an extensive database of airborne in situ microphysical measurements has been constructed and analyzed. Qualitatively, it is first obtained that there is a remarkable stability in the shape of the normalized PSD for the normalized diameters $D_{\mathrm{eq}} / D_{m}$ smaller than 2 , and a larger variability for larger diameters. A global analysis has therefore been conducted in order to assess the errors introduced on radar- and lidar-related parameters (reflectivity, specific attenuation, visible extinction) and cloud parameters (ice water content, effective radius, terminal fall velocity) derived by the use of a single analytical PSD shape for all the PSDs in a large in situ database instead of the "true" shape of each normalized PSD of the database. Different analytical shapes have been evaluated in this way. It has been obtained that the so-called modified gamma shape could be used as an accurate approximation of the normalized PSD for any normalized ice particle size distribution, and for any instrumental or cloud parameter to be derived from the normalized PSD, because it has the unique advantage of well fitting the particular "S shaped" structure of the ice cloud PSDs, where most data points are located. This normalized modified gamma shape $\left[\Gamma_{m}(\alpha, \beta)\right]$ can be expressed as 


$$
\frac{N\left(D_{\mathrm{eq}}\right)}{N_{0}^{*}}=F_{\alpha, \beta}\left(\frac{D_{\mathrm{eq}}}{D_{m}}\right)=\beta \frac{\Gamma(4)}{4^{4}} \frac{\Gamma\left(\frac{\alpha+5}{\beta}\right)^{4+\alpha}}{\Gamma\left(\frac{\alpha+4}{\beta}\right)^{5+\alpha}}\left(\frac{D_{\mathrm{eq}}}{D_{m}}\right)^{\alpha} \exp \left\{-\left[\frac{D_{\mathrm{eq}}}{D_{m}} \frac{\Gamma\left(\frac{\alpha+5}{\beta}\right)}{\Gamma\left(\frac{\alpha+4}{\beta}\right)}\right]^{\beta}\right\},
$$

where $\alpha$ and $\beta$ can be variationally adjusted to the measured PSDs. A value of $\alpha=-1$ and $\beta=3$ produced the smallest errors in Delanoë et al. (2005), with a weak bias and a small standard deviation of $-0.66 \%$ and $4.75 \%$, respectively. These results are still valid when we change the density-diameter relationship.

\section{REFERENCES}

Brown, P. R. A., and P. N. Francis, 1995: Improved measurements of the ice water content in cirrus using a total-water evaporator. J. Atmos. Oceanic Technol., 12, 410-414.

Delanoë, J., A. Protat, J. Testud, D. Bouniol, A. J. Heymsfield, A. Bansemer, P. R. A. Brown, and R. M. Forbes, 2005: Statistical properties of the normalized ice particle size distribution. J. Geophys. Res., 110, D10201, doi:10.1029/2004JD005405.

Donovan, D. P., and A. C. A. P. van Lammeren, 2001: Cloud effective particle size and water content profile retrievals using combined lidar and radar observations, 1, Theory and examples. J. Geophys. Res., 106, 27 425-27 448.

European Space Agency, 2004: EarthCARE_Earth Clouds, Aerosols and Radiation Explorer. The Six Candidate Earth Explorer Missions, ESA/ESTEC Rep. ESA SP-1279(1), 60 pp.

Francis, P. N., A. Jones, R. W. Saunders, K. P. Shine, A. Slingo, and Z. Sun, 1994: An observational and theoretical study of the radiative properties of cirrus: Some results from Ice'89. Quart. J. Roy. Meteor. Soc., 120, 809-848.

Hogan, R. J., D. P. Donovan, C. Tinel, M. E. Brooks, D. Bouniol, A. J. Illingworth, and J. P. V. Poiares Baptista, 2005: Independent evaluation of the ability of spaceborne radar and lidar to retrieve the microphysical and radiative properties of ice clouds. J. Atmos. Oceanic Technol., 23, 211-227.

—, M. P. Mittermaier, and A. J. Illingworth, 2006: The retrieval of ice water content from radar reflectivity factor and temperature and its use in evaluating a mesoscale model. J. Appl. Meteor. Climatol., 45, 301-317.

Illingworth, A. J., and Coauthors, 2007: Cloudnet-Continuous evaluation of cloud profiles in seven operational models using ground-based observations. Bull. Amer. Meteor. Soc., 88, 883-898.

Khvorostyanov, V. I., and J. A. Curry, 2002: Terminal velocities of droplets and crystals: Power laws with continuous parameters over the size spectrum. J. Atmos. Sci., 59, 1872-1884.

Marshall, J. S., and W. Palmer, 1948: The distribution of raindrops with size.. J. Meteor., 5, 165-166.

Matrosov, S. Y., A. V. Korolev, and A. J. Heymsfield, 2002: Profiling cloud ice mass and particle characteristic size from Doppler radar measurements. J. Atmos. Oceanic Technol., 19, 1003-1018.

Mie, G., 1908: Beitrage zur optik truber mieden, speziell kolloidaler metallosingen. Ann. Phys., 25, 377-445.

Mitchell, D., 1996: Use of mass- and area-dimensional power laws for determining precipitation particle terminal velocity. J. Atmos. Sci., 53, 1710-1723.

- and A. J. Heymsfield, 2005: Refinements in the treatment of ice particle terminal velocities, highlighting aggregates. J. Atmos. Sci., 62, 1637-1644.

Okamoto, H., S. Iwasaki, M. Yasui, H. Horie, H. Kuroiva, and H. Kumagai, 2000: An algorithm for retrieval of cloud microphysics using 95-GHz cloud radar and lidar. J. Geophys. Res., 108, 4226, doi:10.1029/2001JD001225.

Protat, A., and Coauthors, 2003: Terminal fall velocity and the FASTEX cyclones. Quart. J. Roy. Meteor. Soc., 129, 15131535.

, J. Delanoë, D. Bouniol, A. J. Heymsfield, A. Bansemer, and P. Brown, 2007: Evaluation of ice water content retrievals from cloud radar reflectivity and temperature using a large airborne in situ microphysical database. J. Appl. Meteor. Climatol., 46, 557-572.

Stephens, G. L., 2005: Cloud feedbacks in the climate system: A critical review. J. Climate, 18, 237-273.

- , and Coauthors, 2002: The CloudSat Mission and the ATrain: A new dimension of space-based observations of clouds and precipitation. Bull. Amer. Meteor. Soc., 83, 17711790.

Tinel, C., J. Testud, J. Pelon, R. H. Hogan, A. Protat, J. Delanoë, and D. Bouniol, 2005: The retrieval of ice cloud properties from cloud radar and lidar synergy. J. Appl. Meteor., 44, 860875.

Wang, Z., and K. Sassen, 2002: Cirrus cloud microphysical property retrieval using lidar and radar measurements. Part I: Algorithm description and comparison with in situ data. $J$. Appl. Meteor., 41, 218-229. 